\title{
Laparoscopic sphincter-saving surgery for low rectal cancer through marker meeting approach
}

\author{
Xuefei Yang ${ }^{1}$, Guixi Zhang ${ }^{1}$, Li Jiang ${ }^{1}$, Hao Zhang ${ }^{1}$, Zhihai Liu ${ }^{1}$, Jingsi Liu ${ }^{1}$, Yang Deng ${ }^{1}$, Kai Pan ${ }^{2}$, \\ Joe King-Man Fan ${ }^{1,3}$ \\ ${ }^{1}$ Division of Gastrointestinal Surgery, Department of Surgery, The University of Hong Kong-Shenzhen Hospital, Shenzhen 518053, China; \\ ${ }^{2}$ Department of Gastrointestinal Surgery, Shenzhen People's Hospital \& Second Clinical Medical College of Jinan University, Shenzhen 518020 , \\ China; ${ }^{3}$ Department of Surgery, Li Ka Shing Faculty of Medicine, The University of Hong Kong, Hong Kong, China \\ Correspondence to: Joe King-Man Fan, MBBS, MS(HKU), FRCSEd. Department of Surgery, The University of Hong Kong-Shenzhen Hospital, \\ No. 1 Haiyuan 1st Road, Futian District, Shenzhen 518053, China. Email: drjoefan@hku.hk.
}

\begin{abstract}
Laparoscopic low anterior resection (LAR) with sphincter preservation for ultra-low rectal cancer is always a challenging operation in colorectal surgery. To achieve negative margins, reducing the difficulty and risks of the procedure are major goals for us. The marker meeting approach we reported can help to accomplish this goal. The key technique for the marker meeting approach is to ensure a clear distal margin in a low resection of the rectum by transanal dissection. This procedure allows access to the space around the distal rectum and mesorectum and to pack the gauzes in the distal part of the space as a landmark. Routine laparoscopic LAR was performed to dissect the space until reaching the gauzes packed above and achieve complete mobilization of the rectum and mesorectum. This surgical procedure is simpler and reduces the difficulty of the operation. Therefore, it is expected to reduce the risk of surgery-related complications and positive margins and is suitable to be widely applied and extended in clinical practice. The short-term and long-term clinical outcomes of the marker meeting approach need more research in large samples.
\end{abstract}

Keywords: Low rectal cancer; marker meeting; laparoscopic low anterior resection (laparoscopic LAR); total mesorectal excision (TME)

Submitted May 16, 2018. Accepted for publication Jul 24, 2018.

doi: $10.21037 /$ atm.2018.08.02

View this article at: http://dx.doi.org/10.21037/atm.2018.08.02

\section{Introduction}

Total mesorectal excision (TME) first described in 1982 by Heald $e t$ al. has become the gold standard of surgical treatment for rectal cancer (1). Laparoscopic procedure for rectal cancer has shown short- and long-term oncological efficacies similar to open surgery (2-4), and it has the advantages of early recovery of bowel function, less blood loss and postoperative pain with shorter hospitalization (4-6). TME performed laparoscopically has been carried out worldwide (7-9). Laparoscopic radical low resection of low rectal cancer with sphincter preservation has been one of the most difficult procedures in colorectal surgery. If the tumor is located less than $6 \mathrm{~cm}$ from the anal verge, especially in male patients with high BMI, it is difficult to expose and dissect in the narrow pelvic cavity and transect the rectum using linear staplers. Multiple stapling is the main contributing factor of anastomotic leakage after surgery by double-stapling approach $(10,11)$. The operative difficulties also contribute to the risk of positive distal margin and circumferential margin $(12,13)$ which affects the quality of surgery and the rate of anal sphincter preservation. Transanal total mesorectal excision (TaTME) is an alternative approach developed to solve these problems, but it is a difficult procedure, requires specific equipments and instruments, and is available only in a few experienced centers. To explore a safe and effective minimally invasive treatment of low rectal cancer with anal sphincter preservation and reduce the difficulty of the procedure, we report a new surgical technique, called the marker meeting approach. This 
procedure can increase the quality and radicularity of tumor resection and meanwhile reducing the operative difficulty of low anterior resection with anal sphincter preservation. To the best of our knowledge, there is no public report of this procedure for TME.

\section{Case presentation}

\section{Patient's demographics}

A 64-year-old male with history of hypertension on antihypertensive presented with 6-month history of per rectal bleeding. Digital rectal examination revealed a cauliflowerlike tumor located in the right posterior wall at $5 \mathrm{~cm}$ from the anal verge. Colonoscopy revealed an ulcerated tumor 5-9 $\mathrm{cm}$ to the anal verge. Histological examination confirmed the diagnosis of rectal adenocarcinoma. In addition, a total of 14 colonic polyps (3-12 $\mathrm{mm}$ in size) were removed during colonoscopy, and the pathological examination confirmed tubular adenoma. Enhanced abdominal computed tomography (CT) showed a rectal irregular tumor and multiple small lymph nodes in the mesorectum. Enhanced pelvic magnetic resonance imaging (MRI) showed an irregular rectal tumor $5 \mathrm{~cm}$ from the anal verge and located in the right posterior wall of the rectum involving $1 / 2$ of the rectal circumference. The tumor's length was $38 \mathrm{~mm}$ from the proximal to the distal rectum, and its thickness was appropriately $12 \mathrm{~mm}$. Continuously enhanced rapid scanning showed four or five small lymph nodes of $5-10 \mathrm{~mm}$ in diameter in the rectal mesentery, suggesting lymph node metastases. Chest radiography was unremarkable. The clinical stage was T3N1M0. After assessment by a multidisciplinary team, including colorectal surgeons, oncologists and radiologists, planed for preoperative neoadjuvant chemoradiotherapy. The regimen for this chemoradiotherapy was as follows: pelvis, rapid arc, 50 Gy/45 Gy/25 Frs to PTV 50/45, 34 days, Xeloda $1,500 \mathrm{mg}$, twice daily (5 days/weekly). Per rectal bleeding subsided after the 12 th radiotherapy session. Post neoadjuvant chemoirradiation (6 weeks) enhanced pelvic MRI showed a significantly reduced rectal tumor and slightly irregular thickening of local rectal mucosa. The maximum thickness of the mucosa was $7 \mathrm{~mm}$, and the thickening mucosa was significantly enhanced with contrast. The previously noted lymph nodes had subsided. On the 58th day after the end of chemoradiotherapy, sigmoidoscopy revealed an ulcer, $5.5 \mathrm{~cm}$ to the anal verge, which involved $1 / 4$ of the rectal circumference. Digital rectal examination revealed a rigid and moveable tumor located in the right posterior wall, $5.5 \mathrm{~cm}$ to the anal verge. Laparoscopic low anterior resection (LAR) was planned in the 12 th weeks after the completion of chemoradiotherapy (14).

\section{Equipment and consumables}

High definition laparoscopic video system (EVIS EXERA III, CV190, OLYMPUS ${ }^{\circledR}$ ), laparoscopic high flow insufflation unit (UHI-4, OLYMPUS ${ }^{\circledR}$ ), routine laparoscopic instruments including atraumatic graspers, trocars $(5,10$ and $12 \mathrm{~mm}, \mathrm{GZTK}^{\circledR}$ ) and vascular clips (Lapro-Clip ${ }^{\mathrm{TM}}$, COVIDIEN ${ }^{\mathrm{TM}}$ ). Anoscope used in the procedure for prolapse and hemorrhoids (PPH) (FRANKENMAN ${ }^{\circledR}$ ), circular anal retractor (TongLuKangXin ${ }^{\circledR}$ ), Ultrasonic dissector (Thunderbeat, OLYMPUS ${ }^{\circledR}$ ).

\section{Surgical technique}

\section{Preoperative preparation}

Polyethylene glycol $192 \mathrm{~g}$ dissolved in 3,000 mL water was administered to the patient at $8 \mathrm{p} . \mathrm{m}$. the day before the operation for bowel preparation. Prophylactic antibiotics (cefuroxime $1.5 \mathrm{~g}$ and metronidazole $500 \mathrm{mg}$ ) were administered intravenously $0.5 \mathrm{~h}$ before the surgical procedure. After anesthesia, urethral catheter was inserted and the patient was positioned in a lithotomy position with the thighs laid flat paralleled to the floor. Abdominal operative site was disinfected with alcohol and chlorohexidine solution. The rectum and the perianal area were irrigated and disinfected with iodophor. The patient was then placed in a $30^{\circ}$ Trendelenburg position.

\section{Diagnostic laparoscopy}

According to the conventional laparoscopic approach, a $10-\mathrm{mm}$ trocar was placed below the umbilicus by open technique under direct vision. The laparoscope was placed into the abdominal cavity to confirm the absence of intraabdominal metastasis and other anomalies that may have affected the surgical decision.

\section{Transanal purse-string suture under the cancer}

The patient's position was changed from the $30^{\circ}$ Trendelenburg position to routine lithotomy position. An anal dilator and semicircular anoscope, instruments used in $\mathrm{PPH}$, were used for the procedure. A purse-string suture was performed $1 \mathrm{~cm}$ below the tumor. The suture was held with hemostat forceps after ligation (Figure 1). 


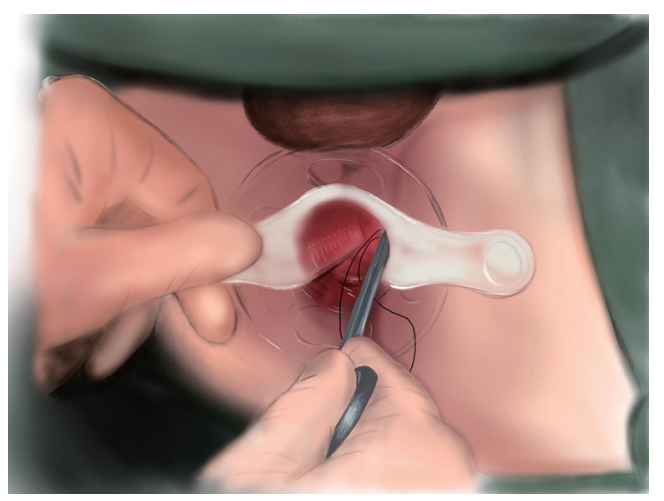

Figure 1 Purse-string suture below the tumor through anus (animated drawing: Dr. Jingsi Liu).

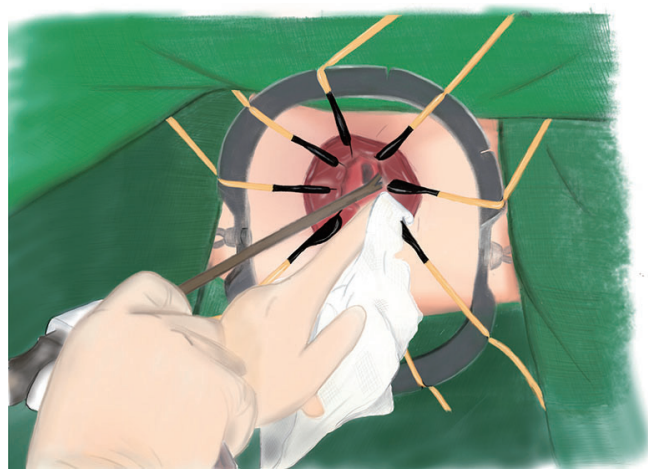

Figure 2 Transanal full-thickness rectal resection with ultrasonic dissector (animated drawing: Dr. Jingsi Liu).

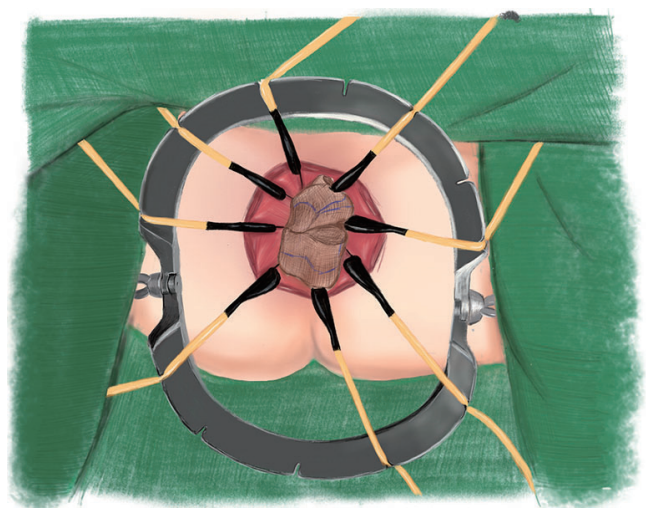

Figure 3 Gauze packing into anus (animated drawing: Dr. Jingsi Liu).

\section{Transanal full-thickness rectal resection}

The semicircular anoscope and anal dilator were then removed. The circular anal retractor was used for retracting

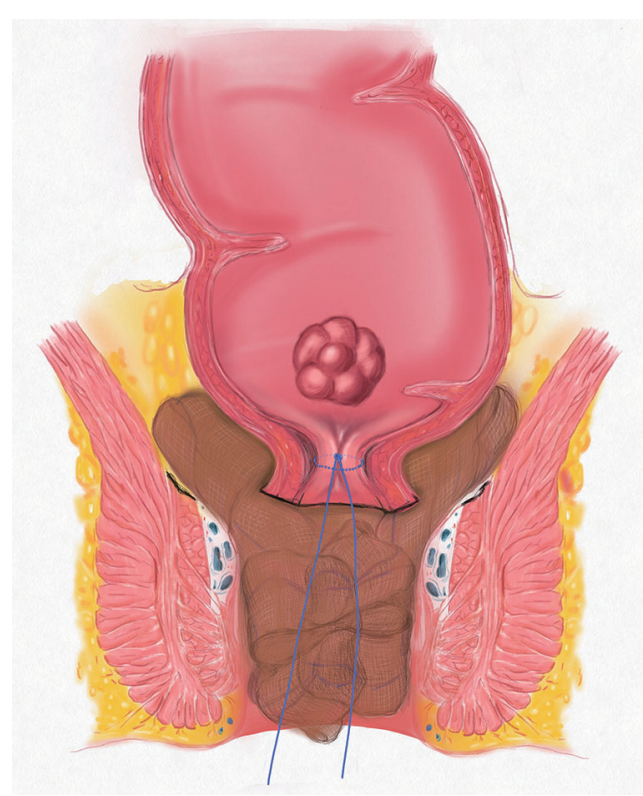

Figure 4 Gauze packing into the space surrounding the rectum and push the rectal stump to the pelvis slightly (animated drawing: Dr. Jingsi Liu).

the anal canal. Ultrasonic dissector was used to incise the mucosa $0.5-1.0 \mathrm{~cm}$ below the purse-string suture and to dissect the full thickness of the rectal wall. The pursestring-sutured rectum was slightly pushed toward the cranial side to confirm that the rectum had been completely transected and that the mesorectal plane was accessible (Figure 2).

\section{Transanal gauzes packing}

Five pieces of iodophor-impregnated gauze $(5 \mathrm{~cm} \times 10 \mathrm{~cm})$ were packed through anus and then the circular anal retractor was removed. The packed gauzes located in the para-rectal space help to push the purse-string sutured rectal stump to the cranial side of pelvis (Figures 3,4).

\section{Laparoscopic TME}

Five trocars were used for performing laparoscopic rectal resection in usual manner (Figure 5). The inferior mesenteric artery was doubly ligated at the root and transected by routine approach. The inferior mesenteric vein was then divided between ligatures in similar manner. The sigmoid colon and the mesentery were separated along the Toldt's space, and the mesentery was dissected from the ligated blood vessels to the distal sigmoid colon. The separation continued along the Toldt's space toward the pelvis to access the presacral space. Along the presacral 


\section{Page 4 of 8}

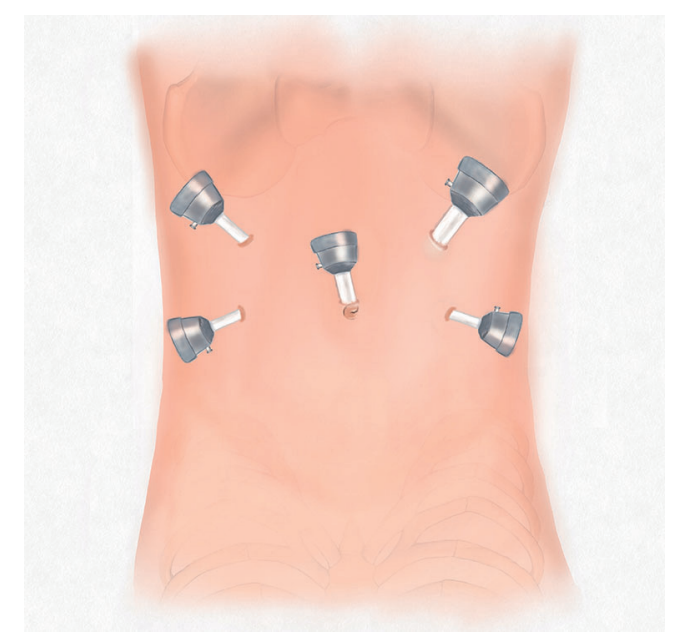

Figure 5 Trocar placement.

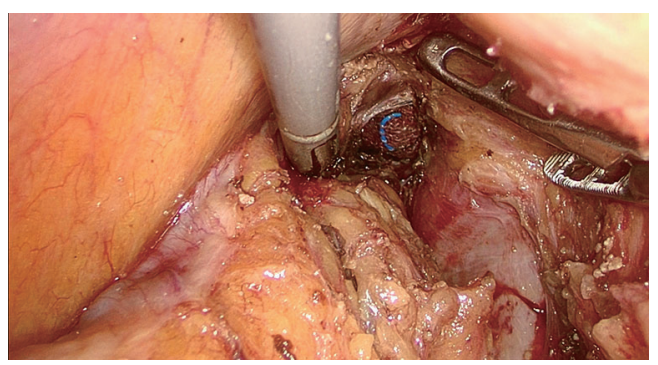

Figure 6 Meeting the gauzes.

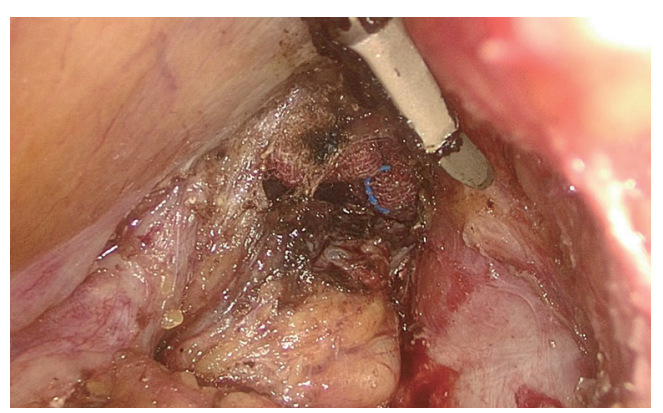

Figure 7 Completely free the purse-string-sutured rectal stump along the surface of the gauzes.

space toward the pelvic floor, the dissection continued to the space posterior to the rectal mesentery and the bilateral space between the rectal mesentery and pelvic side walls. The anterior rectal wall was dissected along with preservation of the Denonvilliers' fascia toward the pelvic floor.

\section{Yang et al. New surgical exploration for low rectal cancer}

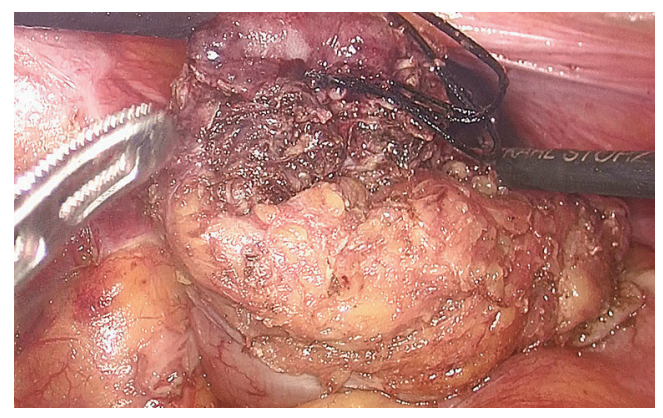

Figure 8 The preserved suture tail in place indicated the reliability of the purse-string suture.

\section{Marker meeting}

During the dissection to the pelvic floor, the visualization of the gauzes indicated approaching the operative space with the gauzes positioned in the para-rectal space from transanal side (Figure 6). At this point, a circumference dissection of the rectum at the level of the gauzes could completely mobilize the purse-string-sutured rectal stump without difficulty (Figure 7). This procedure was named as marker meeting approach. The preserved suture end in place indicated the reliability of the purse-string suture (Figure 8).

\section{Rectal resection through small incision}

The incision over the left lower abdomen for trocar placement was extended to a $4-\mathrm{cm}$ small incision for specimen extraction with wound protection. The sigmoid colon stump, which was closed with purse-string suture, was placed back into the abdominal cavity with the suture tail preserved. The small incision was then closed in layers and the pneumoperitoneum was re-established. The patient's position was adjusted to the general lithotomy position. The circular anal retractor was used to dilate the anal canal to fully expose the dentate line. A laparoscope was used to view the pelvis while removing the gauzes from the anus. Under laparoscopic guidance atraumatic forceps were used to pull the stump of the sigmoid colon by the suture tail out of the anal canal for handsewn anastomosis.

\section{Coloanal anastomosis}

The purse-string suture in the stump of the sigmoid colon was removed. Under direct vision, coloanal anastomosis was performed manually by 4/O Maxon sutures (Figure 9). A pelvic drainage tube was placed through the left lower abdominal wall, and diversion loop ileostomy was created at right lower abdominal wall. At the end of anastomosis, a 


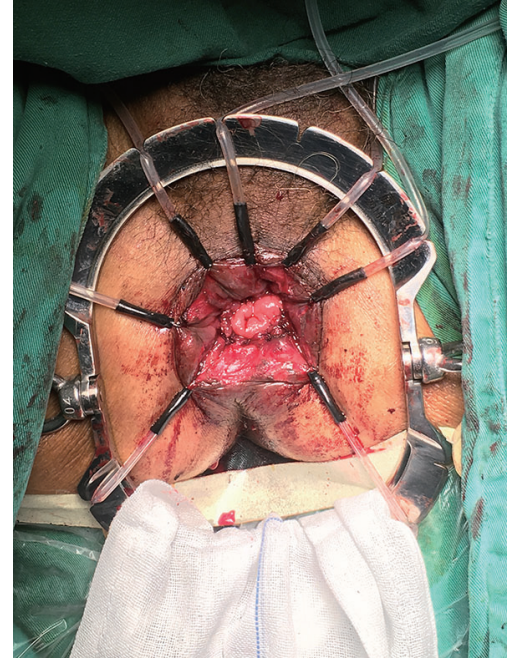

Figure 9 Manually coloanal anastomosis under direct vision.

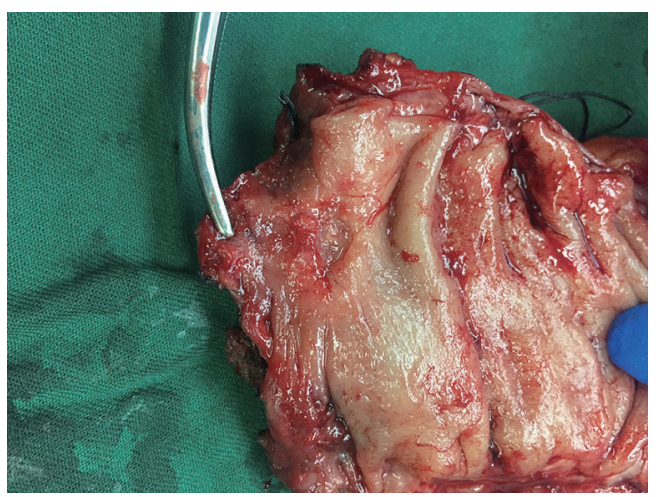

Figure 10 Gross examination of the distal margin of the specimen was approximately $1.5 \mathrm{~cm}$ to the tumor.

drainage tube was placed though anus. All the incisions for trocar were closed. The procedure was completed.

\section{Technical points}

Before the marker meeting approach, iodophor was used in rectal irrigation for disinfection and tumoricidal purposes. The anal dilator and semicircular anoscope of $\mathrm{PPH}$ instruments help to expose the anal canal and the lower rectum and provide direct vision for the purse-string suture at $1 \mathrm{~cm}$ distal to the tumor. At the step using an ultrasonic dissector to transect the distal rectum through the anus, the circular anal retractor is required to adequately retract the anal canal to clearly expose the proposed cutting line and the areola space (mesorectal space) around the distal rectum. At the beginning of the procedure, routine laparoscopic exploration should be carried out prior to the rectum being transected through the anus, this is to rule out some conditions that may affect the surgical plan. The abdominal laparoscopic procedure is the same as conventional laparoscopic LAR. During the operation, the patient's position should be adjusted according to the procedural requirements. The general lithotomy position is used for the operation in the anal canal, while the $30^{\circ}$ Trendelenburg position is used for the abdominal procedure. After the rectum is transected, slight separation in the space around the rectum toward the cranial side may be needed, and the gauzes should be located in the space around the distal rectum to ensure the smooth implementation of the marker meeting approach. During laparoscopic surgery for separation of the distal rectum, an assistant should help to retract the rectum to the cranial side until visualization of the gauzes then the complete separation of the rectal stump and rectal mesentery can be performed safely and simply along the surface of the gauzes. During handsewn coloanal anastomosis, the circular anal retractor is needed to fully dilate the anal canal to facilitate the anastomosis.

\section{Postoperative pathology and recovery}

The operative time was 330 minutes, and the intraoperative blood loss was $30 \mathrm{~mL}$. The quality of TME was complete (Grade I) according to Magnetic Resonance Imaging and Rectal Cancer European Equivalence Study criteria, and the distal margin of the specimen was $1.5 \mathrm{~cm}$ to the tumor (Figure 10). The pathological report is as follows. The ulcerative mass in the rectum was a moderately differentiated adenocarcinoma invading muscularis propria (pT2); lymphovascular and perineural invasion was absent; and the resection margins including circumferential margins were clear. No carcinoma was seen in 10 lymph nodes from perirectal fat tissue and two lymph nodes from the fat tissue around the root of the inferior mesenteric artery. The patient recovered uneventfully after the surgery. Fluid diet and normal diet were started on postoperative day 1 and day 4, respectively. Diversion loop ileostomy started to function with flatus and stool output on post-operation day 2. The anal drainage tube was removed at $48 \mathrm{~h}$ and no drainage was seen. The pelvic drainage amount was ranging from 5 to $215 \mathrm{~mL} /$ day. The color of the drainage gradually changed to clear from the straw color seen on the first day after surgery. The drainage amount gradually decreased, 
and the drainage tube was removed on the 19th day after surgery. The treatment plan for the first year from the oncologist suggested follow-up every 3 months. One week after surgery, digital rectal examination was performed and anastomosis was intact with reduction of the contractility of the anal sphincter. Another digital rectal examination was performed 70 days after surgery and confirmed the normal contractility of the anal sphincter. Flexible sigmoidoscopy on 71 days after surgery confirmed intact anastomosis without stenosis.

\section{Discussion}

To solve the difficult problems of laparoscopic radical resection of low rectal cancer with sphincter preservation, TaTME emerged. In 2010, Lacy performed the world's first laparoscopic-assisted TaTME (15). In 2013, Zhang et al. (16) from China and Leroy et al. (17) from France each reported two patients undergoing total TaTME. By 2017, the TaTME Registry Collaborative had reported 720 cases of this procedure from 66 centers in 23 countries, concluding that TaTME appears to be an oncologically safe and effective technique with acceptable shortterm patient outcomes and good specimen quality (18). However, the TaTME is a technically demanding procedure and requires special instruments. So far, it has been carried out only in a few experienced centers. The limited number of reported cases also suggests the difficulty of applying and extending the procedure in clinical practice. How to simply and safely perform laparoscopic radical resection of low rectal cancer with sphincter preservation has been a major goal of colorectal surgery.

During laparoscopic sphincter-saving surgery for low rectal cancer, the main problem is how to safely transect the low rectum to ensure distal negative margin for the colorectal or coloanal anastomosis. The marker meeting approach reported here can be a better solution to this problem. Rectal irrigation and closure with a pursestring suture distal to the tumor is in line with the tumor contamination-free principle. Transection of the rectum distal to the purse-string suture can ensure a negative margin. With use of a circular anal retractor for exposure, it is not difficult to transect the distal rectum through the anal canal under direct vision. This approach can avoid the difficulty of transecting the low rectum in the narrow pelvis from the abdominal side and the risk of complications caused by multiple stapling-bleeding, breakdown of staples, etc. After transection of the distal rectum, dissection continues in mesorectal plane then the purse-string-sutured rectal stump was pushed toward the cephalad side to place the gauzes in the pararectal space. The gauzes serve as a landmark during laparoscopic dissection. The abdominal procedure is the same as conventional laparoscopic LAR, including ligation of the inferior mesenteric vessels and mobilization of the sigmoid colon and its mesentery. In the pelvis, the dissection should follow TME principle toward the pelvic floor. During this procedure, visualization of the gauzes indicates that the complete dissection of the rectum and rectal mesentery which can be safely performed by dissection at the level of the surface of the gauzes. After transection and removal of specimen via the abdominal wound, the sigmoid stump is closed with a purse-string suture, and the suture tail is retained. Under laparoscopic guidance, the gauzes were removed from the anal canal, and the sigmoid stump can be easily retracted out of the anal canal. With the use of the circular anal retractor for exposure, the manually sutured anastomosis under direct vision is not difficult to perform. Our patient in this report was a male patient with a long anal canal $(3 \mathrm{~cm})$. Preoperative sigmoidoscopy and digital anal examination showed that the tumor was $5.5 \mathrm{~cm}$ from the anal verge. A purse-string suture was performed $1 \mathrm{~cm}$ below the tumor. The rectum was transected $1 \mathrm{~cm}$ distal to the purse-string suture. At the last step, the colon was anastomosed to the dentate line. Diversion loop ileostomy was created to prevent septic complications from anastomotic leakage (19).

Dr. Pan of our federal team began to perform the marker meeting approach in Shenzhen People's Hospital, Shenzhen, China dated back in 2014 and published his technique in a Chinese surgical book in 2016 (20). The case reported in this work is the first marker meeting approach in the University of Hong Kong-Shenzhen Hospital, Shenzhen, China. To the best of our knowledge, this type of procedure (transecting the distal rectum through anus while using gauzes as landmark and to facilitate the operations) has not been reported in international journals so far. The operative technique of marker meeting approach is totally different to that of perineal transanal approach reported previously (21) because of the use of the gauze as a marker and the facilitating advantage. The core technique of the marker meeting approach is to ensure a negative margin during transection of the distal rectum via the anal canal after closure of the rectum distal to the tumor by purse-string suture. The gauzes packed via the anal canal are served as a landmark that can be visualized during laparoscopic dissection. The gauzes can also push the rectal stump 
cranially slightly that can facilitate the dissection in deep pelvic cavity. Isolated dissection via the abdominal cavity or the anal canal can simplify the operation in the lower pelvis. The indication for the marker meeting approach supposed to be low rectal cancer without external sphincter invasion. Theoretically, even for the cases need inter-sphincteric resection (ISR), marker meeting approach is expected to be performed after obtaining inter-sphincteric plane and purse-string of the distal margin.

According to updated reports, the overall complication rate of TaTME is up to $40.3 \%$, and the rate of postoperative major complications is up to $11.5 \%$ (22). In contrast, the marker meeting approach does not require special instruments or a transanal pneumorectum. The operations via the anal canal only include simple transection of the rectum and slight dissection of the space around the rectum and can be performed under direct vision. The abdominal surgery is the same as in conventional laparoscopic LAR. Therefore, the specific complications arise from TaTME, including retroperitoneal or mesenteric emphysema, ureteral injury and bleeding, can be avoided. The marker meeting approach can safely and simply solve the problem of anal sphincter preservation in the surgery of low rectal cancer. However, this procedure requires further validation by large scale cohort or randomized trial to prove its safety and efficacy.

\section{Conclusions}

The key innovations in the marker meeting approach include transanal resection of the lower rectum and use of gauzes packing via the anal canal as a landmark that can be visualized for the meeting of the dissection space between transanal procedure and abdominal procedure. This surgical procedure is simpler than TaTME and laparoscopic LAR and reduces the difficulty of the operation. Therefore, it is expected to reduce the risk of surgery-related complications and positive margins. The marker meeting approach is a new type of procedure that is different from TaTME and laparoscopic LAR. It is expected to solve the difficulties and drawbacks of laparoscopic LAR in the lower pelvic area. Moreover, it is simpler and expectedly safer than TaTME. We believe that the procedure is more applicable and can be widely extended in practice. The short-term and long-term clinical outcomes of the marker meeting approach requires further validation by large scale cohort or randomized trial to prove its safety and efficacy.

\section{Acknowledgements}

None.

\section{Footnote}

Conflicts of Interest: The authors have no conflicts of interest to declare.

Informed Consent: Institutional Review Board in our center waived the approval process and the informed consent of case video for single patient if it contains surgical procedures or techniques with no individual identifier and intended risk for individual patient.

\section{References}

1. Heald RJ, Moran BJ, Ryall RD, et al. Rectal cancer: the Basingstoke experience of total mesorectal excision, 19781997. Arch Surg 1998;133:894-9.

2. Pędziwiatr M, Małczak P, Mizera M, et al. There is no difference in outcome between laparoscopic and open surgery for rectal cancer: a systematic review and metaanalysis on short- and long-term oncologic outcomes. Tech Coloproctol 2017;21:595-604.

3. Arezzo A, Passera R, Salvai A, et al. Laparoscopy for rectal cancer is oncologically adequate: a systematic review and meta-analysis of the literature. Surg Endosc 2015; 29:334-48.

4. Lujan J, Valero G, Biondo S, et al. Laparoscopic versus open surgery for rectal cancer: results of a prospective multicentre analysis of 4,970 patients. Surg Endosc 2013;27:295-302.

5. Guillou PJ, Quirke P, Thorpe H, et al. MRC CLASICC trial group. Short-term endpoints of conventional versus laparoscopic-assisted surgery in patients with colorectal cancer (MRC CLASICC trial): multicentre, randomised controlled trial. Lancet 2005; 365:1718-26.

6. Jayne DG, Guillou PJ, Thorpe H, et al. Randomized trial of laparoscopic-assisted resection of colorectal carcinoma: 3-year results of the UK MRC CLASICC Trial Group. J Clin Oncol 2007;25:3061-8.

7. Jin K, Wang J, Lan H, et al. Laparoscopic surgery for colorectal cancer in China: an overview. Int J Clin Exp Med 2014;7:4635-45.

8. Askari A, Nachiappan S, Currie A, et al. Selection for laparoscopic resection confers a survival benefit in colorectal cancer surgery in England. Surg Endosc 
2016;30:3839-47.

9. Surgical Care and Outcomes Assessment Program (SCOAP) Collaborative, Kwon S, Billingham R, et al. Adoption of laparoscopy for elective colorectal resection: a report from the Surgical Care and Outcomes Assessment Program. J Am Coll Surg 2012; 214:909-18.e1.

10. Park JS, Choi GS, Kim SH, et al. Multicenter analysis of risk factors for anastomotic leakage after laparoscopic rectal cancer excision: the Korean laparoscopic colorectal surgery study group. Ann Surg 2013;257:665-71.

11. Kawada K, Hasegawa S, Hida K, et al. Risk factors for anastomotic leakage after laparoscopic low anterior resection with DST anastomosis. Surg Endosc 2014;28:2988-95.

12. van der Pas MH, Haglind E, Cuesta MA, et al. Laparoscopic versus open surgery for rectal cancer (COLOR II): short-term outcomes of a randomised, phase 3 trial. Lancet Oncol 2013;14:210-8.

13. Battersby NJ, How P, Moran B, et al. Prospective Validation of a Low Rectal Cancer Magnetic Resonance Imaging Staging System and Development of a Local Recurrence Risk Stratification Model: The MERCURY II Study. Ann Surg 2016;263:751-60.

14. Foster JD, Jones EL, Falk S, et al. Timing of surgery after long-course neoadjuvant chemoradiotherapy for rectal cancer: a systematic review of the literature. Dis Colon Rectum 2013;56:921-30.

Cite this article as: Yang X, Zhang G, Jiang L, Zhang H, Liu Z, Liu J, Deng Y, Pan K, Fan JK. Laparoscopic sphincter-saving surgery for low rectal cancer through marker meeting approach. Ann Transl Med 2018;6(16):324. doi: 10.21037/atm.2018.08.02
15. Sylla P, Rattner DW, Delgado S, et al. NOTES transanal rectal cancer resection using transanal endoscopic microsurgery and laparoscopic assistance. Surg Endosc 2010;24:1205-10.

16. Zhang H, Zhang YS, Jin XW, et al. Transanal single-port laparoscopic total mesorectal excision in the treatment of rectal cancer. Tech Coloproctol 2013;17:117-23.

17. Leroy J, Barry BD, Melani A, et al. No-scar transanal total mesorectal excision: the last step to pure NOTES for colorectal surgery. JAMA Surg 2013;148:226-30; discussion 231.

18. Penna M, Hompes R, Arnold S, et al. Transanal Total Mesorectal Excision: International Registry Results of the First 720 Cases. Ann Surg 2017;266:111-7.

19. Hanna MH, Vinci A, Pigazzi A. Diverting ileostomy in colorectal surgery: when is it necessary? Langenbecks Arch Surg 2015;400:145-52.

20. Pan K, Yang X. Laparoscopic Gastrointestinal Surgery. Beijing: People's Medical Publishing House, 2016.

21. Denost Q, Adam JP, Rullier A, et al. Perineal transanal approach: a new standard for laparoscopic sphincter-saving resection in low rectal cancer, a randomized trial. Ann Surg 2014;260:993-9.

22. Deijen CL, Tsai A, Koedam TW, et al. Clinical outcomes and case volume effect of transanal total mesorectal excision for rectal cancer: a systematic review. Tech Coloproctol 2016;20:811-24. 